\title{
Densidade de substratos dependendo dos métodos de análise e níveis de umidade
}

\author{
Maria Helena Fermino'; Atelene N Kämpf² \\ ${ }^{1}$ FEPAGRO, R. Gonçalves Dias 570,90130-060 Porto Alegre-RS; maria-fermino@fepagro.rs.gov.br; ${ }^{2}$ nkampf@cpovo.net
}

\begin{abstract}
RESUMO
O termo "substrato para plantas" refere-se ao meio de crescimento usado no cultivo em recipientes, freqüentemente resultante da mistura de materiais. A qualidade final depende das propriedades físicas e químicas de cada componente e da interação entre eles, as quais precisam ser caracterizadas. Apesar de obras referenciais serem unânimes em incluir a densidade volumétrica na caracterização de substratos, ocorrem dificuldades para interpretar laudos emitidos por diferentes laboratórios, porque estes utilizam métodos diferentes ou variações de um mesmo método. Além das diferenças entre os métodos, podem ocorrer outras causas de variação na obtenção dos resultados de uma mesma amostra. O presente trabalho visa demonstrar duas das causas de variação na determinação da densidade volumétrica: 1) o método de análise [método do duplo anel (CEN), método utilizado pela indústria (IND) e método da autocompactação (AUTO)] dos substratos pó de coco, areia de rio média lavada, turfa fibrosa in natura e Plantmax ${ }^{\circledR}$ Hortaliça; 2) e o nível inicial de umidade $(0,62,0,69$ e $0,72 \mathrm{~g} / \mathrm{g})$ em amostras de turfa fibrosa in natura. Constatou-se que os valores de densidade, determinados pelo método AUTO, representam cerca de $20 \%$ a mais do que os valores determinados pelo método IND; as relações entre os métodos AUTO e CEN, bem como CEN e IND não são uniformes, variando conforme o material; a umidade inicial da amostra também representa causa de variação nos resultados.
\end{abstract}

Palavras-chave: autocompactação, duplo anel.

\begin{abstract}
Density of substrates depending on the analytical methods and the levels of humidity

The term "substrate for plants" refers to the growth medium used in cultivation in containers, often resulting from the mixture of materials. The final quality depends on the physical and chemical attributes of each component and the interaction among them, which need to be characterized. Although the reference works are unanimous including volumetric density for substrate characterization, the interpretation of reports from different laboratories is difficult, because they use different methods or variations of the same method. In addition to the differences between methods, there might be other causes of variation in the achievement of results from a single sample. This work aims to demonstrate two of the causes of variation in the determination of density by volume: 1) the method of analysis [method of double ring (CEN), the method used by the industry (IND) and method of auto compact (AUTO) of substrate dust] of coconut, washed sand of river, peat brown in natura and Plantmax ${ }^{\circledR}$ for horticulture; 2) and the initial level of humidity (0.62, 0.69 and $0.72 \mathrm{~g} / \mathrm{g}$ ) in in natura samples of peat brown. The density values, determined by the AUTO, represented about $20 \%$ more than the values determined by IND; relations between the AUTO and CEN methods as well as CEN and IND were not uniform, depending on the material. Initial moisture of the sample also represents the cause of variation in the results.
\end{abstract}

Keywords: self-compacting, double ring.

(Recebido para publicação em 16 de novembro de 2010; aceito em 23 de janeiro de 2012)

(Received on November 16, 2010; accepted on January 23, 2012)

$\mathrm{O}$ termo "substrato para plantas" refere-se ao meio de crescimento usado no cultivo em recipientes. É um meio poroso, formado por partículas sólidas e poros. As partículas sólidas, de origem mineral, orgânica ou sintética podem variar muito em aspectos físicos como aparência, forma, tamanho e massa específica.

As empresas produtoras de "substrato para plantas" nem sempre se localizam próximo ao mercado consumidor e o transporte a grandes distâncias onera o preço, limitando, com freqüência, sua aquisição. Para reduzir custos gerais, não raro o interessado elabora seu próprio substrato, misturando materiais disponíveis na região. Elaborar uma mistura de qualidade não é uma tarefa fácil pois, a qualidade final depende de cada componente e da interação entre eles. Para conhecer a qualidade de um material, é necessário determinar suas propriedades físicas e químicas. Fonteno (2007, 1993) ressalta a importância de conhecer as propriedades físicas dos componentes de substratos, o que permitirá direcioná-los para as necessidades da planta. Da mesma forma, dirigindo-se a pesquisadores, afirma que protocolos de pesquisa com substratos devem incluir mensurações das propriedades físicas e hidráulicas.

A caracterização das misturas e/ ou das matérias primas é, portanto, de fundamental importância para conhecer suas propriedades e definir seu uso e manejo. Entre as propriedades físicas destaca-se a densidade (bulk density ou densidade volumétrica), correspondente à relação entre a massa e o volume total de uma amostra (volume total $=$ volume de sólidos + volume de poros da amostra). Assim, a densidade integra a relação das características exigidas pelo Ministério da Agricultura, Pecuária e Abastecimento (MAPA, 2004) a serem informadas no rótulo dos produtos comerciais registrados no país.

Os valores da densidade volumétrica de materiais usados na composição de substratos são expressos em $\mathrm{kg} / \mathrm{m}^{3}$, unidade oficial conforme o Sistema Internacional. A literatura técnica para produção de plantas em recipientes refere-se à densidade volumétrica, muitas vezes, na unidade em $\mathrm{g} / \mathrm{L}$, cujo valor 
numérico é o mesmo que em $\mathrm{kg} / \mathrm{m}^{3}$.

O conhecimento do valor da densidade volumétrica tem várias aplicações, como no cultivo em recipientes, servindo como parâmetro para o manejo da irrigação. $\mathrm{Na}$ análise de nutrientes (com referência à massa da amostra), a densidade é indispensável para a interpretação dos laudos e recomendações práticas. Também, auxilia na seleção do recipiente a ser utilizado, conforme especificado por Kämpf (2000): misturas muito leves são próprias para bandejas, enquanto as de alta densidade são mais adequadas para recipientes maiores.

Outros estudos sobre análises de "substrato para plantas" são unânimes em incluir a densidade volumétrica na caracterização de um produto ou material (Gabriels \& Verdonck, 1991; Wever \& Eymar, 1999; MAPA, 2004, 2006, 2007). Mas, apesar da concordância generalizada, Fonteno (1993) nos Estados Unidos e Baumgarten (2002) na Áustria, relatam a ocorrência de dificuldades para interpretar laudos emitidos por diferentes laboratórios, do mesmo país, porque os laboratórios utilizam métodos diferentes ou variações de um mesmo método para análises (Günther, 1984; Waller \& Wilson, 1984; Miner, 1994).

Corroborando, Vence et al. (2011), determinando a densidade de diferentes substratos com diferentes métodos, entre eles aquele sugerido pela norma européia (EN-13041) e aquele descrito na legislação brasileira (IN 31) afirmam que, em parte, as diferenças encontradas nos resultados da densidade podem ser explicadas pelo tratamento inicial da amostra nos métodos.

Além das diferenças entre os métodos, podem ocorrer outras causas de variação na obtenção dos resultados de uma mesma amostra. Burés (1997) e Kämpf et al. (1999) referem-se a alterações na densidade de um substrato devido à força empregada no empacotamento da amostra em determinado volume. O presente estudo tem como objetivo analisar duas das causas de variação na determinação da densidade volumétrica: 1) os métodos de análise; 2) o nível inicial de umidade das amostras e suas implicações práticas no uso do substrato.

\section{MATERIAL E MÉTODOS}

Os estudos foram realizados em laboratórios da FEPAGRO e da UFRGS, em Porto Alegre (RS).

Experimento 1 - Comparação entre métodos para determinar a densidade volumétrica de substratos

Os materiais utilizados representam componentes isolados: pó de coco (PC) da empresa Amafibra ${ }^{\circledR}$, Pará; areia de rio média lavada (A); turfa fibrosa in natura (TF) da empresa Florestal ${ }^{\circledR}$, Araranguá, Santa Catarina, e o produto Plantmax ${ }^{\circledR}$ Hortaliça da empresa Eucatex ${ }^{\circledR}$, que representa uma mistura à base de casca de pinus. Para as análises, foram selecionados três métodos da literatura internacional, já praticados no Brasil e que se diferenciam pela forma de medir o volume da amostra em um dado recipiente. Originalmente, os métodos utilizam recipientes de diferentes dimensões, o que resulta em amostras de volumes diferentes. Entretanto, para permitir a comparação direta entre os resultados, optou-se por uniformizar o volume das amostras nos referidos procedimentos, estabelecendo um "recipiente-padrão". Utilizou-se, para tanto, um conjunto de cilindros de Policloreto de Vinila (PVC), conforme a Figura 1. Cada conjunto é formado por duas unidades de cano de PVC (100 mm de diâmetro e $100 \mathrm{~mm}$ de comprimento, com volume interno de $750 \mathrm{~mL}$ ). O cilindro inferior é fechado na base por uma tampa (cap) do mesmo material; na parte superior deste cilindro colocou-se uma conexão móvel em forma de anel (luva), que serve para fixar o segundo cilindro sobre o primeiro, de forma similar ao sugerido por Verdonck (1984).

Neste experimento utilizou-se delineamento inteiramente casualizado, com três repetições.

1) IND - baseado nos procedimentos praticados pelas indústrias de substratos, no mercado nacional e internacional, onde o volume do produto é calibrado no equipamento de preencher a embalagem, sem pressão de compactação (não existe referência em artigos científicos, no entanto, é tema conhecido entre técnicos. A variação pode se dar no volume utilizado). Cada amostra foi peneirada sobre o recipiente-padrão, de forma a deixá-la cair livremente no recipiente, até alcançar a altura da metade do cilindro superior. Posteriormente foram retirados o anel e o cilindro superior e, cuidando para não pressionar a amostra, sendo que sua superfície superior foi levemente aplanada conforme os limites do cilindro inferior, posteriormente, procedeu-se a pesagem.

2) CEN - baseia-se no Método do Duplo Anel, recomendado pelo Comitê Europeu de Normatização (Aendekerk et al., 2000; Training Course, 2003). O recipiente padrão foi preenchido com a amostra, de forma similar ao método anterior, sem pressão, até a metade do cilindro superior. Sobre a superfície da amostra, no cilindro superior, foi colocado um peso de $687,50 \mathrm{~g}$, por um período de 180 segundos. Após este período, o anel e o cilindro superior foram retirados, nivelando-se a superfície da amostra conforme os limites do cilindro inferior, pesando-se a seguir.

3) AUTO - Método da autocompactação, com base no método VDLUFA, adotado na Alemanha (Röber \& Schaller, 1985) e Áustria (Baumgarten, 2002; Training Course, 2003). É o método oficial adotado pelo MAPA (MAPA, 2004) e está assim descrito: “A proveta plástica de $500 \mathrm{~mL}$ deverá ser preenchida até aproximadamente a marca de 300 $\mathrm{mL}$ com o substrato na umidade atual. Em seguida, esta proveta é deixada cair, sob a ação de sua própria massa, de uma altura de $10 \mathrm{~cm}$, por 10 (dez) vezes consecutivas. Com auxílio da espátula nivela-se a superfície levemente e lê-se o volume obtido (mL). Em seguida, pesa-se o material (g) descontando a massa da proveta. $\mathrm{O}$ procedimento deverá ser repetido por três vezes com subamostras diferentes. Deverá ser expresso o valor da média das medições, em número inteiro."

Para a determinação da densidade seca, amostras do material úmido são levadas à estufa para secagem a $65^{\circ} \mathrm{C}$ até peso constante (ou 48 horas) (deve-se expressar o valor médio de três aferições, em número inteiro).

Os valores de densidade úmida (Du), matéria seca (MS) (amostra seca a $65^{\circ} \mathrm{C}$ até peso constante) e densidade seca (Ds) foram obtidos aplicando-se as 
seguintes fórmulas:

$\mathrm{Du}\left(\mathrm{kg} / \mathrm{m}^{3}\right)=1000$ (massa úmida*/ Volume**)

Umidade gravimétrica $(\mathrm{g} / \mathrm{g} /)=($ massa úmida* - massa seca*)/massa úmida* trica

MS $(\mathrm{g} / \mathrm{g})=1$ - Umidade gravimé-

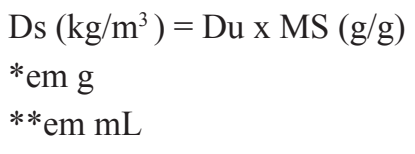

As análises estatísticas foram realizadas com auxílio do Programa SAS e os resultados de Ds e Du foram submetidos à análise de variância e, quando significativo, as diferenças entre as médias foram comparadas através do teste de Tukey em nível de 1\% de significância.

Experimento 2 - Influência da umidade inicial da amostra sobre a determinação da densidade seca

Amostras de Turfa Fibrosa in natura (TF), similar ao material usado no estudo anterior, foram preparadas em crescentes níveis de umidade e submetidas aos três métodos de análise referidos no Experimento 1, em delineamento inteiramente casualisado em esquema fatorial 3 x 3 (métodos de análise x níveis de umidade inicial da amostra) com três repetições. Os níveis de umidade nas amostras foram obtidos com ajuda de um aspersor manual.

Para a caracterização dos níveis, as amostras foram colocadas na palma da mão, fechando-se os dedos com pressão e abrindo-se a seguir. Nível 1: ao ser comprimida contra a palma da mão, a amostra mantinha-se aglutinada, porém separando-se com facilidade ao abrir a mão; nível 2: ao ser comprimida, a amostra, sem desprender água entre os dedos, formava torrão coeso no centro da mão; nível 3: ao ser comprimida, a amostra formava torrão e escorriam gotas de água entre os dedos.

Para a determinação da umidade gravimétrica, duas subamostras de 100 g (massa úmida), de cada nível, foram secas a $65^{\circ} \mathrm{C}$ até peso constante. Os resultados indicam os valores de 62, 69 e $72 \%$ de umidade gravimétrica equivalente a $0,62,0,69$ e $0,72 \mathrm{~g} / \mathrm{g}$ para os níveis 1,2 e 3 , respectivamente.

Para a determinação da densidade seca, amostras do material úmido são levadas à estufa para secagem a $65^{\circ} \mathrm{C}$ até peso constante (ou 48 horas) (deve-se expressar o valor médio de três aferições, em número inteiro).

Os valores de densidade úmida (Du), matéria seca (MS) (amostra seca a $65^{\circ} \mathrm{C}$ até peso constante) e densidade seca (Ds) foram obtidos aplicando-se as seguintes fórmulas:

$\mathrm{Du}\left(\mathrm{kg} \mathrm{m}^{-3}\right)=1000$ (massa úmida*/ Volume**)

Umidade gravimétrica $(\mathrm{g} / \mathrm{g})=$ (massa úmida* - massa seca*)/massa úmida*

MS $(\mathrm{g} / \mathrm{g})=1$ - Umidade gravimétrica

$$
\begin{aligned}
& \text { Ds }\left(\mathrm{kg} / \mathrm{m}^{-3}\right)=\text { Du x MS }(\mathrm{g} / \mathrm{g}) \\
& * \text { em } \mathrm{g} \\
& * * \text { em mL }
\end{aligned}
$$

As análises estatísticas foram realizadas com auxílio do Programa SAS e os resultados de Ds e Du foram submetidos à análise de variância e quando significativo as diferenças entre as médias foram comparadas através do teste de Tukey em nível de 1\% de significância.

\section{RESULTADOS E DISCUSSÃO}

\author{
Experimento 1 - Comparação \\ entre métodos para determinar a
}

densidade volumétrica de substratos

Os dados demonstram variações significativas nos valores de densidade úmida e seca dos quatro materiais, conforme os métodos testados $(\mathrm{p}>0,01)$ (Tabela 1).

Ao submeter os materiais a procedimentos de análises que se distinguem especialmente na intensidade da pressão de compactação da amostra num recipiente padronizado, esperava-se que os resultados fossem proporcionais à pressão de compactação relativa a cada método. Assim, formulou-se inicialmente a hipótese de que os valores de densidade deveriam ser menores em IND (sem compactação), intermediários em CEN (compactação na parte superior da amostra) e mais altos em AUTO (autocompactação ao longo de todo o perfil da amostra no recipiente).

Os resultados obtidos, entretanto, confirmaram apenas parcialmente a hipótese formulada pois, os materiais analisados mostraram comportamentos diferentes entre si. O pó de coco representa o material mais leve entre os componentes analisados neste estudo. Os valores de sua densidade seca (65

Tabela 1. Valores absolutos da densidade seca e úmida de pó de coco, areia de rio e turfa fibrosa e dos métodos de determinação de densidade IND, CEN e AUTO e, em percentual, tomando por base os valores obtidos no método IND (absolute values of wet and dry density of powder coconut, river sand and peat, and methods for determining density of IND, CEN and AUTO and, as a percentage, based on the values obtained in IND method). Porto Alegre, FEPAGRO, 2007.

\begin{tabular}{ccccc}
\hline \multirow{2}{*}{ Métodos } & \multicolumn{4}{c}{ Materiais (densidade seca (g/L)) } \\
\cline { 2 - 5 } & Pó de coco & Areia de rio & Turfa fibrosa & Plantmax $^{\circledR}$ \\
\hline IND & $54 \mathrm{~b}$ & $1209 \mathrm{~b}$ & $175 \mathrm{~b}$ & $238 \mathrm{~b}$ \\
CEN & $65 \mathrm{a}$ & $1204 \mathrm{~b}$ & $194 \mathrm{ab}$ & $263 \mathrm{ab}$ \\
AUTO & $65 \mathrm{a}$ & $1455 \mathrm{a}$ & $209 \mathrm{a}$ & $278 \mathrm{a}$ \\
\hline \multicolumn{5}{c}{ Densidade úmida (g/L) } \\
\hline IND & $252 \mathrm{~b}$ & $1466 \mathrm{~b}$ & $479 \mathrm{c}$ & $544 \mathrm{~b}$ \\
CEN & $307 \mathrm{a}$ & $1460 \mathrm{~b}$ & $532 \mathrm{~b}$ & $601 \mathrm{ab}$ \\
AUTO & $306 \mathrm{a}$ & $1764 \mathrm{a}$ & $572 \mathrm{a}$ & $635 \mathrm{a}$ \\
\hline \multicolumn{5}{c}{} \\
\hline IND & Densidade seca e úmida (\% sobre IND) \\
CEN & 100 & 100 & 100 & 100 \\
AUTO & 122 & 100 & 111 & 110 \\
\hline
\end{tabular}

Letras diferentes, na coluna, indicam diferença estatística significatica pelo teste de Tukey a $1 \%$ (different letters in column, indicate statistical significant difference by Tukey test $1 \%$ ); IND - utilizado pela indústria de substrato; CEN - método do duplo anel e AUTO autocompactação (IND-substrate used by industry; CEN-method of double ring and AUTO - Auto Compact). 


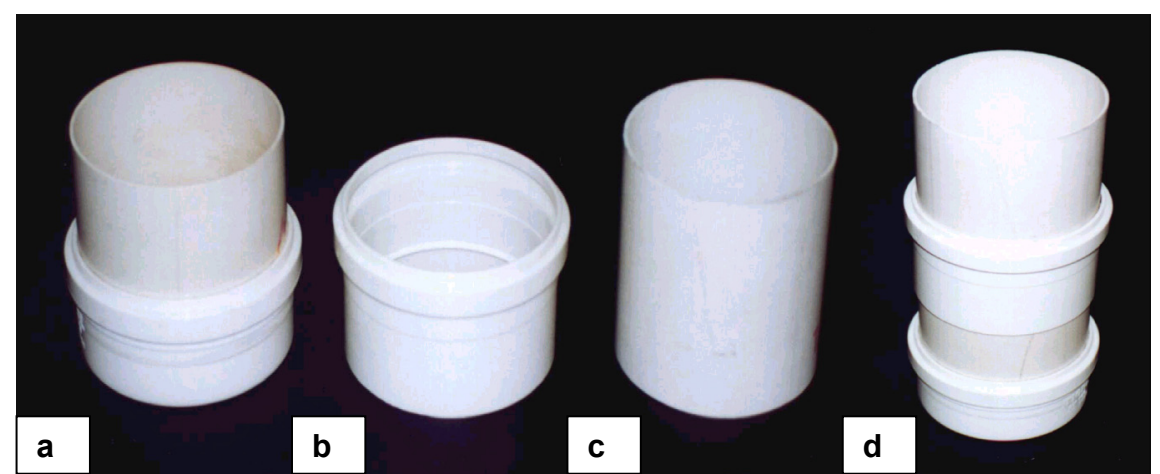

Figura 1. Cilindros de policloreto de vinila (PVC) utilizados para determinação da densidade: a) "cap" + cilindro com volume interno de $750 \mathrm{~mL}$; b) "luva" para conexão entre os cilindros; c) cilindro com volume interno de $750 \mathrm{~mL}$ para sobrepor ao primeiro; d) conjunto pronto para efetuar a determinação da densidade (polyvinyl chloride cylinders (PVC) used for determination of density: a) "cap" + cylinder with internal volume of $750 \mathrm{~mL}$; b) "glove" for connection between the rollers; c) cylinder with internal volume of $750 \mathrm{~mL}$ to overlay the first; d) set ready to make the determination of density). Porto Alegre, UFRGS, 2003.

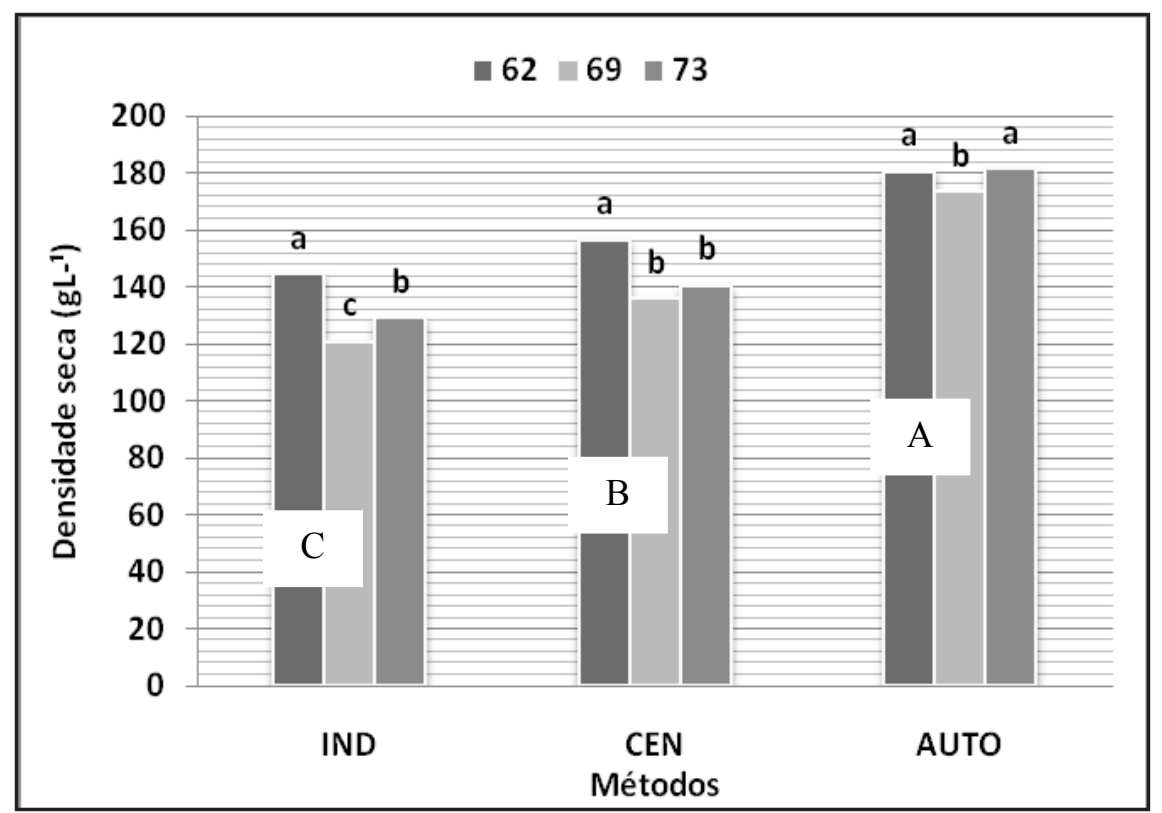

Figura 2. Densidade seca da turfa fibrosa in natura submetida a três níveis de umidade (62, 69 e $72 \%$ ) e três métodos de determinação da densidade (IND, CEN e AUTO) [dry density of fibrous peat in natura subjected to three levels of moisture (62, 69 and 72\%) and three methods for determining the density (IND, CEN and AUTO)].

Médias seguidas pela mesma letra, maiúscula ou minúscula, não diferem entre si pelo teste de Tukey a $5 \%$ de probabilidade (average values followed by the same letter, uppercase or lowercase, do not differ by Tukey test 5\% probability). Porto Alegre, UFRGS, 2003.

$\mathrm{g} / \mathrm{L}$ ) e úmida (cerca de $307 \mathrm{~g} / \mathrm{L}$ ) não diferem, quando avaliados pelos métodos AUTO ou CEN, porém são menores 54 e $252 \mathrm{~g} / \mathrm{L}$, Ds e Du, respectivamente, se avaliados pelo método IND $(\mathrm{p}<0,01)$. A areia representa o material mais pesado entre os analisados. Os valores de sua densidade seca (cerca de $1200 \mathrm{~g} / \mathrm{L}$ ) e úmida (cerca de $1460 \mathrm{~g} / \mathrm{L}$ ) não diferem, quando avaliados pelos métodos IND e CEN, porém, são maiores (1455 e $1764 \mathrm{~g} / \mathrm{L}$, respectivamente Ds e Du) se avaliados pelo método AUTO $(p<0,01)$. Plantmax ${ }^{\circledR}$ Hortaliça e turfa fibrosa mostraram comportamento intermediário entre o pó de coco e a areia, sendo a hipótese inicial integralmente confirmada apenas nos resultados de densidade úmida de turfa fibrosa (valores obtidos em IND são inferiores aos obtidos em
CEN, que são inferiores aos obtidos em AUTO, $p \leq 0,01)$.

Para quantificar a intensidade da variação provocada pelos métodos testados, os dados absolutos de densidade foram transformados em valores percentuais, tomando-se como base (100\%) os resultados encontrados no método IND, por representarem as mais baixas densidades determinadas.

Comparando os métodos AUTO e IND, todos os valores obtidos no método da autocompactação (AUTO) resultaram em cerca de $20 \%$ a mais sobre o procedimento da indústria (IND). Porém, comparando os métodos CEN e IND, as diferenças não seguem um padrão uniforme e variaram de acordo com o tipo de material analisado.

Considerando-se que os três métodos de determinação da densidade úmida e seca, reproduzem na sua metodologia, três procedimentos usados para encher recipientes de cultivo com substrato em um viveiro de plantas e as variações demonstradas entre os métodos indicam alterações que podem ocorrer na prática, conforme a maneira usada para preencher os recipientes. Por exemplo: um dado substrato é colocado em vasos de 15 a $20 \mathrm{~cm}$ de altura, com capacidade para um litro ou mais de volume, através do procedimento similar ao da autocompactação (deixando-se cair o vaso com substrato algumas vezes, de uma altura vertical próxima a $10 \mathrm{~cm}$ ). Através deste procedimento, espera-se que a quantidade de substrato colocada no vaso contenha cerca de $20 \%$ a mais em partículas sólidas, se comparado ao mesmo substrato colocado em bandejas multicelulares, através do procedimento sem compactação (similar ao método IND). A diferença na quantidade proporcional de sólidos resultante de tais procedimentos deve ter efeitos sobre as demais propriedades físicas daquele substrato, tais como a razão de vazios (relação entre poros e sólidos), porosidade total, espaço de aeração, capacidade de retenção de água e penetrabilidade para as raízes, medida como o valor de impedância mecânica, como demonstrada por Fermino \& Kämpf (2005), além de influenciar na interpretação de análises químicas do substrato, principalmente quando se considera o método 
"Teor Total de Sais Solúveis" (Röber \& Schaller, 1985), onde leva-se em conta a massa do substrato.

Experimento 2 - Influência da umidade inicial da amostra sobre a determinação da densidade seca

Neste estudo, confirma-se a influência do fator Métodos como causa de variação na determinação da densidade de substratos. De forma similar ao observado para a turfa no Experimento 1, os resultados mostram o padrão esperado (valores mais baixos em IND, intermediários em CEN e mais altos em AUTO) com significativas diferenças entre os três procedimentos de análise (Figura 2). A análise estatística revela que o fator Níveis de Umidade também atuou como causa de variação, não apenas da densidade úmida, o que era esperado pelo acréscimo de água na amostra, mas também na densidade seca, havendo interação significativa entre os métodos de determinação e os níveis de umidade na amostra.

Diferenças entre os valores de densidade determinados pelos métodos CEN e AUTO já foram observadas por Baumgarten (2002), que encontrou alta correlação entre os dois procedimentos de análise, calculando que o método CEN represente apenas $75 \%$ do valor determinado pelo método da autocompactação, referido pelo autor como Austrian Standard. Da mesma forma, no presente estudo foi observado que as densidades determinadas em CEN foram $16 \%$ menores no nível $1,29 \%$ no nível 2 e $27 \%$ no nível 3 de umidade quando comparado com as densidades obtidas através do AUTO.

$\mathrm{O}$ valor da densidade seca pode ser tomado como um indicativo da massa dos sólidos presentes na amostra analisada. Neste estudo, observa-se, como regra geral que as amostras da turfa fibrosa preparadas com o mais baixo nível de umidade gravimétrica $(0,62$ $\mathrm{g} / \mathrm{g}$ ) continham mais massa de sólidos do que as amostras do mesmo material preparadas com mais umidade (Figura 2). Conforme Bailey et. al. (2000), a água adicionada a materiais orgânicos, como a turfa, aumenta o volume das partículas, por hidratação das mesmas e consequentemente, ocorreu a formação de mais espaço poroso na amostra. Este fato tem importância prática em viveiro de plantas, especialmente no plantio em bandejas multicelulares (produção em plugs): no momento de preencher os recipientes com o substrato, pode-se reduzir a quantidade de sólidos nas células, aumentando-se o nível da umidade do substrato. Os autores, com ampla experiência na produção em plugs, consideram fundamental molhar o substrato à base de turfa até atingir $67 \%$ de umidade $(0,67 \mathrm{~g} / \mathrm{g})$ no cultivo em bandejas, no mínimo duas horas antes de envasá-lo.

O presente trabalho está de acordo com os resultados obtidos por Baumgarten (2002): o método da autocompactação (AUTO) é simples, rápido e prático, enquanto o método do duplo anel (CEN) consome mais tempo, sendo limitado para uso em rotinas de laboratório.

Portanto, conclui-se que o método de determinação da densidade e o teor de umidade inicial de uma amostra representam causa de variação na obtenção dos resultados de densidade.

\section{AGRADECIMENTOS}

Ao CNPq e às empresa Amafibra ${ }^{\circledR}$, Floresta $l^{\circledR}$ e Eucatex ${ }^{\circledR}$ pela oferta dos substratos utilizados neste trabalho.

\section{REFERÊNCIAS}

AENDEKERK THGL; AENDEKERK GL; CEVAT H; DOLMANS N; ELDEREN C; KIPP JA; KREIJ C; SONNEVELD C; VERHAGEN JBGM; WEVER G 2000. International Substrate Manual. Doetinchem: Elsevier International Business. 94p.

BAILEY DA; FONTENO WC; NELSON. P. 2000, agosto. Greenhouse substrates and fertilization. Disponível em: http://www. ces.ncsu.edu/depts/hort/floriculture/plugs/ ghsusfert.pdf.

BAUMGARTEN A. 2002. Methods of chemical and physical evaluation of substrates for plants. In: FURLANI AMC. Caracterização, manejo e qualidade de substratos para produção de plantas. Campinas: Instituto Agronômico, 122p. (Documentos IAC, 70).

BURÉS, S. 1997. Sustratos. Madrid: Ediciones Agrotécnicas. 341p.

FERMINO MH; KÄMPF A. 2005. Considerations about the packing density of growing media prepared under increasing levels of humidity. Acta Horticulturae 697:147-151.

FONTENO WC. 1993. Problems \& considerations in determining physical properties of horticultural substrates. Substrates in
Horticulture. Acta Horticulture 342:197-204. FONTENO WC. 2007, 20 de julho. A common misconception about substrates. Disponível em: http://www2.ncsu.edu/unity/lockers/ project/hortsublab/pdf/Grower_Article. PM.pdf.

GABRIELS R; VERDONCK O. 1991. Physical and chemical characterization of plant substrates: Towards an European standardization. Acta Horticulturae 294: $249-259$

GÜNTHER J. 1984. Analytics of substrates and problems by transmitting the results into forticultural practice. Acta Horticulturae 150:33-40.

KÄMPF AN. 2000. Produção comercial de plantas ornamentais. Porto Alegre: Gênesis. $254 \mathrm{p}$.

KÄMPF AN; HAMMER PA; KIRK T. 1999. Effect the packing density on the mechanical impedance of root media. Acta Horticulturae 481: 689-694.

MINISTÉRIO DAAGRICULTURA, PECUÁRIA E ABASTECIMENTO. Instrução Normativa $\mathrm{N}^{\mathrm{o}} 14$, de 15 de dezembro de 2004. Publicado no Diário Oficial da União de 15/12/2004, Seção 1, Página 24. Aprova as Definições e Normas sobre as Especificações e as Garantias, as Tolerâncias, o Registro, a Embalagem e a Rotulagem dos Substratos para Plantas, constantes do anexo desta instrução normativa.

MINISTÉRIO DAAGRICULTURA, PECUÁRIA E ABASTECIMENTO. Instrução Normativa $\mathrm{N}^{\circ} 46$, de 12 de setembro de 2006. Publicado no Diário Oficial da União de 14/09/2006, Seção 1, Página 2. Aprova os Métodos Analíticos Oficiais para Análise de Substratos e Condicionadores de Solos.

MINISTÉRIO DAAGRICULTURA, PECUÁRIA E ABASTECIMENTO. Instrução Normativa $\mathrm{N}^{\circ} 17$, de 21 de maio de 2007. Publicado no Diário Oficial da União de 24/05/2007, Seção 1, Página 8. Aprova os Métodos Analíticos Oficiais para Análise de Substratos e Condicionadores de Solos.

MINER AM. 1994. Substratos: propriedades y caracterizacion. Madrid: Ediciones MundiPrens. 172p.

RÖBER R; SCHALLER K. 1985. Pflanzenernährung im Gartenbau. Stuttgart: Ulmer. 352p.

TRAINING COURSE, 2003. CEN - Standards for Chemical and Physical Analysis of Growing Media. ISHS, AGES, Fachhochschule Weihenstephan.

VENCE LB; VALENZUELA OR; SVARTZ HA. 2011. Caractecización físicas de sustratos com fines comerciales: densidad y capacidad de retención de água medidas por diferentes métodos. In: VII Encontro Nacional de Substratos para Plantas. Goiânia: Universidade Federal de Goiás. Anais.

VERDONCK O. 1984. Reviewing and evaluation of new materials used as substrates. Acta Horticulturae 150: 467-473.

WALLER PL; WILSON GCS. 1984. Evaluation of growing media for consumer use. Acta Horticulturae 150: 51-58.

WEVER G; EYMAR E. 1999. Characterisation of the hydrophysical and mechanical properties of pressed blocks for transplanting. Acta Horticulturae 481: 111-119. 